\title{
OPTICAL OBSERVATIONS OF NUCLEI OF GALAXIES
}

\author{
MARIE-HELENE ULRICH \\ Dept. of Astronomy, University of Texas at Austin, Austin, Tex. 78712, U.S.4. \\ and \\ Observatoire de Paris, 92190 Meudon, France
}

\begin{abstract}
Several problems concerning the nuciei of galaxies are particularly interesting at present, either because new data have become available from advances in observational techniques, or else because recent observations suggest that earlier conclusions should be revised. These problems are discussed in this article.

Spectrophotometry in the near infrared shows that the late-type stars in the nucleus of M31 are mostly late giants; the $M / L$ ratio in the nucleus is approximately 15 , a value in agreement with that calculated independently from the velocity dispersion of the stars.

Infrared emission at $10 \mu$ has now been detected in 50 galaxies; the Seyfert-type galaxies are the brightest at $10 \mu$ and there is evidence of variations of the $10 \mu$ flux in NGC 4151, which rules out re-radiation by dust as the mechanism of infrared emission. In each of the galaxies NGC 253 and NGC 3034 an infrared source of dimension $\sim 200 \mathrm{pc}$ is present in the centre which coincides in position and dimension with the central component of the radio source at $1415 \mathrm{MHz}$; since both galaxies are rich in dust and in early-type stars it is plausible that, in these cases, re-radiation by dust is the origin of the infrared emission. Most of the other galaxies detected at $10 \mu$ are spirals with intense and narrow emission lines.

Comparison of the optical and radio properties of nuclei of galaxies show that galaxies with compact nuclear radio sources are more likely to have optical spectra with emission lines than are galaxies without central radio sources. This correlation holds for elliptical and spiral galaxies. Its cause is not understood at present.

The relatively accurate profiles now available for the permitted lines of several Seyfert galaxies show that mass motions are the main broadening agent for these lines. Variations of line intensities have been observed in several Seyfert galaxies. The most likely explanation of these variations is that they are due to the variations of the photoionizing flux; in some cases, however, weakening of the permitted lines could be caused by high-velocity mass motions present in the region emitting the broad component of these lines.

From the investigation of the relatively nearby galaxies it is estimated that at least $5 \%$ of galaxies have expansion motions or non-thermal optical fluxes.
\end{abstract}

\section{Introduction}

The meaning of the term 'galactic nuclei' is evident, at least in a general descriptive sense, yet attempts at defining precisely the nuclei of galaxies immediately meet with difficulties. In practice the nucleus of a galaxy can be defined as the maximum of the surface brightness of the stellar continuum. The nucleus is usually close to or coincident with the centre of symmetry of spiral and elliptical galaxies, the centre of the rotation curve, and the maximum of star density.

At present, the existence of a central object whose limits can be drawn from the surface brightness distribution is not established. The photographs taken by Stratoscope II give a conservative upper limit of 0.08 or $4 \mathrm{pc}$ for the half-power width of the nucleus of NGC 4151 (Schwarzschild, 1971) and show the presence, at the centre of M31, of an elliptical structure with a semi-major axis slightly less than 1 " or $3.3 \mathrm{pc}$ at half-intensity (Danielson et al., 1971). It is not known whether this is a common feature in galactic nuclei. 
Phenomenologically, nuclei of galaxies have an extremely interesting property: in those galaxies where large amounts of energy are released in the form of relativistic particles, thermal and non-thermal radiation and mass motions, these violent events are occurring in or have originated in the nuclei. This fact has an obvious and important consequence which is that the onset of these violent events is linked - in a way which is still unknown - to some of the properties of the nuclei such as, for example, the high stellar density or the strong gravitational field.

Two types of nuclei of galaxies can be clearly distinguished:

(a) quiescent or passive nuclei, in which the optical radiation is emitted by normal stars and a small quantity of interstellar gas.

Much of our knowledge on normal nuclei is based on observations of the nearby spiral galaxies, especially M31, whose nucleus is the most extensively studied at all wavelengths and with the best angular resolution.

(b) active nuclei, for which some of the optical flux is of non-thermal origin and/or is emitted by large clouds of ionized gas. There are extreme cases of active nuclei where the stellar population of the nucleus is undetected, most of the optical flux being of non-thermal origin; the other signs of extreme activity in these nuclei are variations or polarization of the optical flux, infrared or high-frequency radio emission, and mass motions with velocities larger than, say, $1000 \mathrm{~km} \mathrm{~s}^{-1}$. However, it is important to bear in mind that there exists a number of cases where the level of activity of the nucleus is intermediate between that of the quiescent nuclei and that of extremely active nuclei. These intermediate cases display some of the properties of extremely active nuclei, but the total energy involved is smaller. Evidently, low or medium-level activity in the nucleus of a galaxy is difficult to detect, so there is observational selection against mildly active nuclei as compared with extremely active nuclei.

In this paper we discuss problems which are particularly interesting at present, because new data have just become available and are accumulating rapidly due to the advances of observational techniques, or because recent observations yield compelling evidence that earlier conclusions be revised.

The outline of the article is as follows: stellar populations and abundances are discussed in Section 2. Section 3 deals with gas and star motions and Section 4 with dust, interstellar gas and infrared emission. In Section 5, we discuss the observations of profiles and variations of absolute intensity of the permitted lines, and in Section 6 estimate the fraction of galactic nuclei which show signs of activity in the optical range.

\section{Stellar Populations and Abundances}

Most of our knowledge about stellar populations and abundances in nuclei of galaxies comes from narrow-band photometry. As observational techniques develop, the wavelength range which can be observed widens toward the infrared and the ultraviolet, providing new information on the populations of very cool and very hot stars. 
To understand the recent developments in determination of the stellar populations and abundances in galaxies it is useful to recall the three steps from the narrow-band photometry observations to the synthetic model of a stellar population:

(i) choice of a set of narrow bands centred on lines whose equivalent widths are particularly sensitive to stellar types, luminosities, and abundances.

(ii) choice of the types of stars to be included in the population synthesis; the colour indices of the various synthetic models are then compared to the observed colour indices.

(iii) the third step consists of choosing the criteria according to which some of the models will be rejected and others found acceptable. To be astrophysically acceptable, a model has to have at least the two following properties: firstly, a smooth distribution of stars along the main sequence and, secondly, the proportion of any given type of stars must be consistent with the proportions of the two types immediately following and preceding it in stellar evolution.

\subsection{THE NUCleUs OF M31 AT OPTICAL AND NEAR INFRARED WAVELENGTHS}

The nucleus which has been most studied is that of M31, and the extensive observations by Spinrad and Taylor (1971) together with other studies by Wood (1966), McClure and van den Bergh (1968), and McClure (1969) have led to the following results relating to a region $10^{\prime \prime}$ or $33 \mathrm{pc}$ in diameter: (i) the stars are very strong-lined, implying that the abundances of some elements are higher than normal and (ii) there is a strongly dwarf-enriched lower main sequence, indicating that $M / L \approx 44$.

We shall see that recent work confirms the overabundance of some elements but that there is now considerable evidence that the ratio $M / L$ is smaller than 44 and probably close to 15 (However, see the remark on the overabundance in 2.1.1.).

For fitting the computed model indices to those observed, Spinrad and Taylor (1971) employed a trial-and-error method; with this method it is almost unavoidable that some models be overlooked, and Faber (1972) has therefore set up another method based on quadratic programming. The criterion for best fit is that the quantity $\Sigma \sigma^{2}$, which is the sum of the squares of the residuals, be a minimum. Faber applied this method to the data of Spinrad and Taylor with very little alteration. She found that the model obtained by Spinrad and Taylor was not the one giving the minimum value of $\Sigma \sigma^{2}$, and that a model.including a smaller proportion of late $\mathrm{M}$ dwarfs and having $M / L=15$ gave a smaller value of $\Sigma \sigma^{2}$ than theirs. The exclusion of super-metal-rich stars from the ingredients of the synthetic model was found to increase $\Sigma \sigma^{2}$ significantly, confirming the overabundance of some elements in the nucleus of M31.

Two recent observations in the near infrared also indicate that the ratio $\mathrm{M} \mathrm{dwarfs} / \mathrm{M}$ giants must be smaller than was found by Spinrad and Taylor. Whitford (1972 and also this volume p. 169) has observed the nucleus of M31 at the wavelength of the unidentified Wing-Ford band at $9910 \AA$. This band is a useful discriminant of stellar luminosity for types cooler than late $\mathrm{K}$, being much stronger in $\mathrm{M}$ dwarfs than in $\mathrm{M}$ giants. From the observed weakness of this band in the nucleus of M31, Whitford concludes that there is no significant contribution from $\mathrm{M}$ dwarfs at that wavelength. 
The second observation in the infrared is reported by Baldwin et al. (1973) who have observed the absorption band of $\mathrm{CO}$ at $2.3 \mu$. The variations of the intensities of this band with stellar types and luminosities have been studied for a number of stars from G0 to M8. The CO band is very sensitive to the luminosity for stars cooler than late $\mathrm{K}$, being much stronger in giants than in dwarfs. The value of the $\mathrm{CO}$ index measured in the nucleus of M31 (and also of M81) is not consistent with models rich in late-type dwarf stars and this result agrees well with the conclusion drawn by Whitford from the weakness of the Wing-Ford band.

In summary: (i) the re-analysis by Faber of the data obtained by Spinrad and Taylor, (ii) the weakness of the Wing-Ford band as observed by Whitford and (iii) the strength of the $\mathrm{CO}$ band at $2.3 \mu$, all suggest smaller values of the ratio $\mathrm{M}$ dwarf/M giants and of the $M / L$ ratio than in Spinrad and Taylor's best model. The value $M / L=15$ fits well the observed $\mathrm{CO}$ indices in the nuclei of M31 and M81 and agrees with Whitford's observations of M31.

This value of $M / L$ refers to the luminous material only and therefore represents a lower limit to the true value of $M / L$. In Section 3 we see that a recent determination of the velocity dispersion in the nucleus of M31 also leads to a value of $M / L$ close to 15 .

Up to now the CO band has been observed in the nuclei of M31, M81 and NGC 5195 only. If strong $\mathrm{CO}$ bands -implying a large proportion of $\mathrm{M}$ giants - are also observed in giant elliptical galaxies, the cosmological implications would be important because the change with time of the luminosity of a giant-dominated galaxy population is faster than for a population rich in dwarfs; the true value of $q_{0}$ obtained from the magnitude-redshift relation after taking into account a fast rate of luminosity evolution would be smaller than the value which is obtained from the data available now on the stellar content of giant elliptical galaxies (Tinsley, 1973).

\subsubsection{Cautioning Remark on the Overabundance of the Elements}

It has been mentioned above that in the nucleus of M31 the abundance of some elements is higher than normal. When synthesizing populations of galaxies, the abundances of the elements are determined by calculating the number of super-metalrich stars which must be introduced in order that the indices of the synthetic population match the observed indices. The analyses by Spinrad and Taylor (1971) and by Faber (1972) show that in the nucleus of M31 the number of super-metal-rich K giants is about $1 / 50$ the number of $\mathrm{K}$ dwarfs of normal abundances. The expression super-metal-rich stars refers to the specific stars (e.g. $\mu$ Leo, $\phi$ Aur, etc....) which are chosen to be included in the mixture. It must be noted that the super-metal-rich stars constitute the ingredient of the stellar mixture which is known with the least accuracy. In general, in these stars, the elements are overabundant by a factor 4 or less than 4 . These stars do not form an homogeneous class; the overabundance is not the same for all these stars and in a given star it is not the same for all the elements; the exact amounts by which the various elements are overabundant are still a matter of controversy (Strom et al., 1971; Blanc-Vaziaga et al., 1973) and it is not clear how much of the apparent overabundance in these stars is due to atmosphere peculiarities. Sounder 
evidence for overabundance in nuclei of galaxies is provided by the analysis of emission lines from the interstellar gas.

\subsection{THE POPULATION OF HOT STARS - OAO RESULTS}

The effect of a small number of hot stars on the energy distribution at $\lambda>3300 \AA$ has been investigated by Peimbert (1968), Spinrad and Taylor (1971), Tinsley and Spinrad (1971) and Faber (1972). All these authors agree that the presence of a small number of $O$ and B stars ( $\sim 40$ in Spinrad and Taylor's study) is compatible with the narrow-band photometry observations just longward of $3300 \AA$.

Results on 35 galaxies observed by OAO 2 have been presented by Code et al. (1972). The energy distribution shows a minimum near $2400 \AA$ followed by a steep upturn shortward. It does not seem possible to fit the observed curve by any combination of early-type stars since the upturn shortward of $2400 \AA$ is steeper than that produced by the hottest stars. This minimum occurs near the maximum of the galactic interstellar extinction curves.

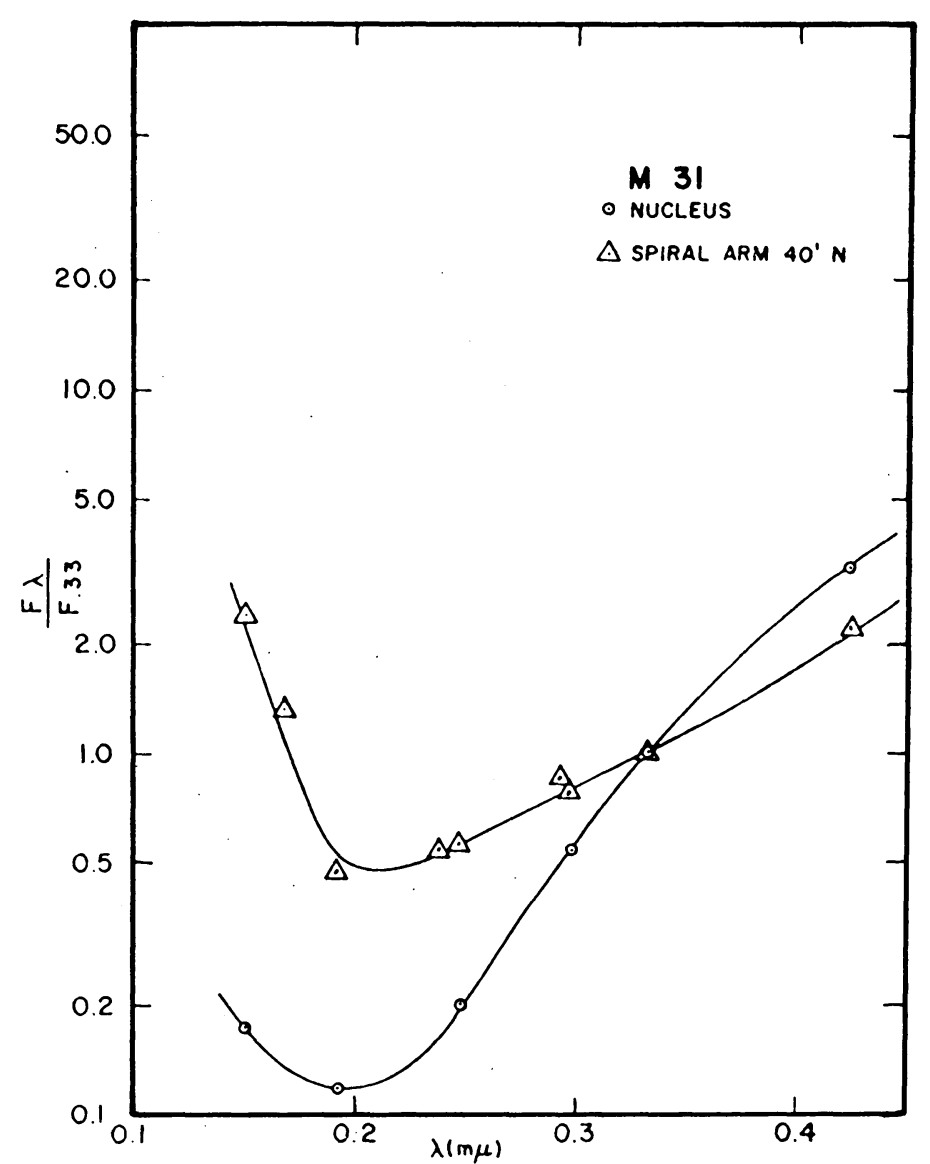

Fig. 1. Energy distribution between 1500 and $4500 \AA$, observed through a $10^{\prime}$ aperture at 2 locations in M31. From Code et al. (1972) 
Figure 1 shows the energy distribution of M31 observed through a 10' aperture (equivalent to $1800 \mathrm{pc}$ in the plane of the galaxy) at two different locations: one centred on the nucleus of $\mathrm{M} 31$, the other centred on a bright super-association $40^{\prime}$ south of the nucleus. It is suggested by Code et al. (1972) that the peculiar shape of the energy distribution between $1500 \AA$ and $3500 \AA$ is due to the presence of earlytype stars whose energy distribution is modified by the interstellar dust present in the galaxies. In view of the similarity between the two extinction curves in Figure 1, the dust particles in the central region and in the spiral arms have at least one property in common, a minimum albedo at $2200 \AA$.

\subsection{VARIATIONS OF STELLAR CONTENT WITH DISTANCE FROM CENTRE IN M31}

The variations of the strengths of several absorption lines with increasing distance from the centre of M31 show that the abundances and probably also the stellar population change from the centre outward. The changes of line strengths and colours occurring within $120^{\prime \prime}$ along the major axis and with a resolution of $10 \AA$ can be summarized as follows:

- the $\mathrm{CN}$ band strength drops sharply with radial distance; the strengths of the $\mathrm{Na}$ D-lines drop off slowly, while the Mg I triplet at $5180 \AA$ shows essentially no change (McClure, 1969; Spinrad et al., 1971).

- the $U-B$ index decreases from 0.79 at the centre to 0.60 at $60^{\prime \prime}$ away then remains constant across the disk. The other colours $B-V, V-R$, and $V-K$ show no change with distance from the centre (Sandage et al., 1969).

The sharp fall of the $\mathrm{CN}$ band just outside the nucleus, combined with the fact that the $\mathrm{Mg}$ I triplet remains constant, implies that the abundances decrease rapidly and also that the ratio $\mathrm{K}$ dwarfs/ $\mathrm{K}$ giants increases outward. The changes in abundance and luminosity must be such that the weakening of the $\mathrm{Mg}$ I triplet due to the decrease of abundance is balanced by the increase of the triplet due to the strong negative luminosity effect (the triplet being much stronger in dwarfs than in giants). The relatively slow weakening of the $\mathrm{Na}$ I D-lines is consistent with the fact that these lines are more sensitive to abundance but have a smaller negative luminosity effect than the Mg I triplet.

A complete set of narrow-band photometric data has been obtained at 1' south of the nucleus of M31 (Spinrad et al., 1971) and analyzed following the procedure used by Spinrad and Taylor (1971) for the nucleus itself. The models with only stars having normal abundances work best at $1^{\prime}$ from the centre of $\mathrm{M} 31$, in contrast to the strong-lined stars required at the nucleus. The best model for the disk has $M / L \approx 45$ as Spinrad and Taylor (1972) had found in the nucleus, but this value may need to be revised when the intensities of the Wing-Ford band and of the $\mathrm{CO}$ band at $2.3 \mu$ are available for this region of the disk.

\subsection{RESULTS FROM NARROW-BAND PHOTOMETRY IN OTHER NEARBY GALAXIES}

The variations of the $\mathrm{CN}$ index with distance from the centre along the major and minor axes of several bright nearby elliptical, S0, Sa and Sb galaxies have been in- 
vestigated by Spinrad et al. (1972) with a $5^{\prime \prime}$ aperture. They found that the $\mathrm{CN}$ index either decreases or stays constant with increasing distance from the centre; they also found that the CN gradient is correlated with the degree of flattening of the central regions of the galaxies, the steepest $\mathrm{CN}$ gradients being found in galaxies which have the steepest gradients of surface brightness. Flattened systems show a lower $\mathrm{CN}$ gradient than do round elliptical galaxies. In NGC 3115, the $\mathrm{CN}$ index appears to be constant along the major axis, whereas along the minor axis it decreases rapidly as in elliptical galaxies.

These observations support the view of Freeman (p. 129) who considers that a galaxy is made up of two components which have had different kinematic and chemical histories - a disk of uniform chemical abundance in centrifugal equilibrium and a bulge which is dynamically relaxed and chemically inhomogeneous.

In the nuclei of the dwarf elliptical galaxies M32, NGC 205 and NGC 5195, the hydrogen lines are particularly strong and the fitting of a synthetic population does not require any super-metal-rich stars (Spinrad, 1972).

Finally it must be remembered that the contribution from a quiescent or mildly active nucleus to the integrated light of a galaxy is negligible. For this reason, the fact that in elliptical galaxies the integrated colours and line strengths are closely correlated with absolute magnitudes at all luminosities (Faber, 1973) reflects differences in large fractions of the total populations rather than differences in the populations of the nuclei alone.

\subsection{Stellar POPUlations in NUClei OF SEyfert galaxies and active galaXies}

The stellar populations in nuclei of Seyfert galaxies cannot be analyzed by narrow-band photometry because of the contamination by the non-stellar continuum and by the emission lines. However, a number of lines or bands of stellar origin have been identified in the spectra of the nuclear regions of several Seyfert galaxies, as listed in Table I.

Alloin et al. (1971) have investigated the stellar populations in the nuclei of some Seyfert and other active galaxies in an attempt to determine the reddening and/or the contribution from non-stellar continuum: they based model stellar populations upon the observed equivalent widths of about 10 absorption lines measured on spectrograms. For a given galactic nucleus, a unique model cannot be determined, but the various models which fit equally well the observed equivalent widths give rise to the same stellar continuum. This calculated continuum is compared with the observed continuum and the difference is interpreted in terms of reddening and/or non-stellar continuum, i.e. thermal emission from gas or non-thermal radiation. This method applied to NGC 1068 (Andrillat and Souffrin, 1971) shows that the reddening determined from an analysis of the stellar continuum in the nuclear region 7 " in diameter is definitely smaller than the reddening measured by Wampler (1971) in the emission line region, using an aperture of 10". In the nucleus of NGC 3031, Alloin et al. (1971) find an ultra-violet non-stellar source which can be readily accounted for by thermal emission. In NGC 2903, the observed continuum near 
TABLE I

Absorption lines and bands of stellar origin observed in the nuclear regions of some Seyfert galaxies

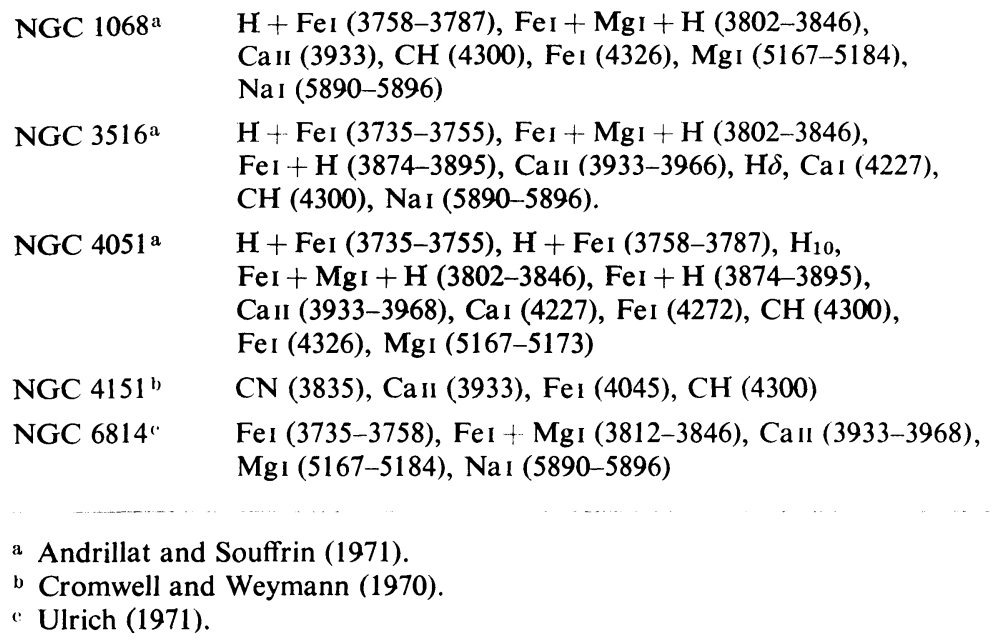

$4000 \AA$ is found to be larger than the sum of the radiation from the $F$ and $G$ stars whose features dominate the spectrum at this wavelength and the thermal bremsstrahlung calculated from the emission line intensities; it is concluded that up to $\sim 30 \%$ of the light at $4000 \AA$ may be of non-thermal origin (Andrillat et al., 1972).

\section{Stars and Gas Motions}

The most important quantity provided by observations of stars and gas motions in nuclei of galaxies is the central mass, obtained from the velocity dispersion of the stars or from the rotation curve measured from emission or absorption lines in a small region surrounding the nucleus. However, there are many cases where the emission lines cannot be used for mass determination: in the spectra of elliptical galaxies the lines are usually very weak or absent, and in some active nuclei the ionized gas has expansion motions and therefore the emission lines do not provide a reliable value of the central mass. In these cases, observations of absorption lines are very desirable, but there are further problems: firstly the lines are difficult to observe outside the nucleus, and secondly the interpretation of the observed rotationcurve is not straightforward because the stellar population in elliptical galaxies and in the central regions of spiral galaxies has a three-dimensional distribution. At a given point on the spectrum the observed velocity results from the combined effect of all the stars along the line of sight; these stars are not all at the same distance from the centre so the features of the rotation curve are blurred. In principle, the determination of the true rotation curve in the equatorial plane from the observed rotation curve requires a knowledge of the spatial distribution of the stars. 
A most important parameter which is needed to interpret the observed velocities is the orientation in space of the equatorial plane of the galaxy; this is usually determined from the position angle of the ellipse which is the projection on the sky of some morphological feature of the galaxy, e.g. isophotes, 'rings' of $\mathrm{H}$ il regions, etc. It is assumed that such features are circular and lie in the equatorial plane of the galaxy, an assumption which is not always valid since different features of the same galaxy sometimes describe ellipses with major axes in different position angles. M31 and NGC 4151 (see below) are examples of galaxies where such ambiguity appears.

As far as non-circular motions are concerned, the observational situation has not changed since the review by Burbidge (1970), except in the case of M31 (see below) and the Seyfert-type galaxies (see Section 5). I summarize it as follows: non-circular motions are known to exist in a dozen of mildly active nuclei; in most cases the data are consistent with expansion motions but are insufficient to enable one to build an accurate model of the geometry and of the velocity field; the mass and physical conditions of the gas involved in the expansion motions are very poorly known.

The major results of recent observations of the motions in the central region of M31 are described below because they illustrate the variety of motions which can be found in the central region of a normal galaxy.

\subsection{Velocity Field in the CENTRAL REgION OF M31}

M31 is the galaxy for which the motions of the stars and gas have been the most extensively studied. The other observations in the X-ray, optical, infrared and radio ranges of wavelength are consistent with M31 being a normal massive spiral galaxy.

In the region $15^{\prime \prime}$ in radius surrounding the nucleus, the stellar motions have been observed by Lallemand et al. (1960) and by Morton and Thuan (1973). The first observations were made in P.A. 37.7 along the major axis of the whole galaxy, and with very good atmospheric seeing. The absorption lines show that the rotation curve reaches a maximum of $80 \mathrm{~km} \mathrm{~s}^{-1}$ at about $2^{\prime \prime}$ or $6 \mathrm{pc}$ from the centre; beyond 2".2, the rotation velocity decreases and reaches a minimum of $40 \mathrm{~km} \mathrm{~s}^{-1}$ at $6^{\prime \prime}$ or $18 \mathrm{pc}$ from the nucleus. The rotation curve obtained from the recent observations (Morton and Thuan, 1973) in the EW direction and with a 4" seeing disk displays qualitatively the same features as those found by Lallemand et al., viz. a maximum of velocity near $3^{\prime \prime}$ from the centre, then a decrease to almost $0 \mathrm{~km} \mathrm{~s}^{-1}$ near $4^{\prime \prime}$. The maximum of velocity is $44 \mathrm{~km} \mathrm{~s}^{-1}$ on the west of the nucleus and $62 \mathrm{~km} \mathrm{~s}^{-1}$ on the east of the nucleus, significantly smaller than the value obtained by Lallemand et al. Morton and Thuan note that, according to Johnson (1961), the nucleus within a $3.3 \times 2$ ".4 isophote has its major axis at P.A. $=52^{\circ} \pm 2^{\circ}$. If this value of the position angle of the major axis is relevant for the small region discussed here, then Lallemand et al.'s observations were made $14^{\circ}$ away from the major axis of the nuclear region, and Morton and Thuan's observations were made $38^{\circ}$ away from the major axis and on the opposite side of the major axis; in these conditions the two sets of observations roughly agree with purely circular motions whereas, if the major axis lies in P.A. 37.7 , the large value of the maximum of the velocity found in the EW direction should 
imply a significant radial expansion of the stars. The velocity dispersion observed by Morton and Thuan is $120 \pm 30 \mathrm{~km} \mathrm{~s}^{-1}$. The $M / L$ ratio in the region $9 \mathrm{pc}$ in radius, calculated from this value of the velocity dispersion, is 13 with an uncertainty by a factor a little less than 2. This agrees well with the value of 15 discussed in Section 2.

An extensive study of the motions of the interstellar gas within $600 \mathrm{pc}$ of the centre has been made by Rubin and Ford (1971). The major conclusions are as follows: (i) the gas is gathered in a very thin rotating disk about $25 \mathrm{pc}$ thick and has a maximum rotational velocity of $200 \mathrm{~km} \mathrm{~s}^{-1}$ at $400 \mathrm{pc}$ from the centre; (ii) expansion motions with velocities up to $100 \mathrm{~km} \mathrm{~s}^{-1}$ occur in a fairly symmetric pattern in P.A. $68^{\circ}-128^{\circ}$ and P.A. $248^{\circ}-278^{\circ}$; (iii) the emission lines are also seen in front of the absorbing dust lanes present in the NE quadrant, showing that there is gas outside the galactic plane. In front of the dust, the lines emitted by ionized gas outside the plane are broader and also show velocities up to $60 \mathrm{~km} \mathrm{~s}^{-1}$ in excess of the rotation velocity; this is tentatively interpreted as gas falling towards the plane.

The same spectrographic material was also used to investigate the stellar motions (Rubin et al., 1973). The maximum rotation velocity observed along the major axis is $140 \mathrm{~km} \mathrm{~s}^{-1}$; this is shown by Rubin et al. to be consistent with the bulk of the stellar population having the same rotation motions as the thin layer of gas. Because the galaxy is viewed only $13^{\circ}$ from edge-on and also because the distribution of the stars is thicker than that of the gas, stars with distances from the centre differing by several hundred parsecs contribute to the absorption lines measured; this integration along the line of sight has the effect of lowering the value of the observed maximum of the rotation curve. Along the far minor axis, the stars appear to have the same expanding motion as is observed in the gas and it is possible (Rubin et al., 1973) that the motion in the area may be linked with the branching of the spiral pattern.

In summary, the following features are observed within $500 \mathrm{pc}$ of the centre of M31:

(i) an elliptical structure with a semi-major axis slightly less than $1^{\prime \prime}$ or $3.3 \mathrm{pc}$ at half-intensity (Danielson et al., 1971);

(ii) a first maximum of the rotation curve at 2.2 or $7 \mathrm{pc}$, measured from the absorption lines;

(iii) a second maximum of the rotation curve at $400 \mathrm{pc}$, measured from the emission lines, the radial velocities of the absorption lines being consistent with this feature;

(iv) expansion motions of the ionized gas in two opposite sectors in the equatorial plane, motions which extend over $400 \mathrm{pc}$ and have velocities $\sim 100 \mathrm{~km} \mathrm{~s}^{-1}$; and

(v) gas outside the equatorial plane which is perhaps falling back to the plane.

\subsection{Velocity DisPERSIONS IN NUCLEI OF GALAXIES}

Determination of the mass of the central region or of the total mass of spheroidal galaxies is possible through the observations of the stellar velocity dispersion $\sigma$, provided several assumptions are made (Poveda, 1958). As observational techniques develop, it is possible to observe $\sigma$ with an increasingly high accuracy. Recently, new determinations of $\sigma$ have been published for 5 elliptical or S0 galaxies (Morton and Chevalier, 1973), for M32 (Richstone and Sargent, 1972), and for the central region 
of M31 (Morton and Thuan, 1973). The results are listed in Table II. The valuès of $\sigma$ from the recent observations are smaller than had been found earlier and consequently lead to lower values of $M / L$. The value of $\sigma$ in Table II were obtained by fitting the spectra observed in the range $4000-4500 \AA$ with artificially broadened spectra of G8III or K0III stars, which contribute most of the light in that range. However, a large fraction of the mass could be contained in fainter low-mass stars which may not have the same $\sigma$ and this is the source of the main uncertainty in the calculation of the masses of nuclei. Only in the case of M31 has the new value of $\sigma$ been used to calculate the mass of the nucleus: within $9 \mathrm{pc}$ from the centre the mass is $(1.8 \pm 0.8) \times 10^{8} M_{\odot}$ (Morton and Thuan, 1973).

\section{TABLE II}

Recent measurements of the velocity dispersions in 7 galactic nuclei

\begin{tabular}{|c|c|c|c|}
\hline NGC & Galaxy type & $\begin{array}{l}\text { Velocity } \\
\text { dispersion } \\
\left(\mathrm{km} \mathrm{s}^{-1}\right)\end{array}$ & $M_{\mathrm{pg}}$ \\
\hline 1889 & E0 & $110 \pm 20^{a}$ & -18.0 \\
\hline 3115 & E7/S0 & $215+20^{a}$ & -18.7 \\
\hline 4473 & E5 & $160 \pm 20^{a}$ & -20.6 \\
\hline 4494 & E1 & $160+20^{a}$ & -19.9 \\
\hline 7332 & So & $160 \pm 20^{\mathrm{a}}$ & -19.6 \\
\hline 221 (M32) & E2 & $60 \pm 8^{b}$ & --15.5 \\
\hline 224 (M31) & $\begin{array}{l}\text { Central region } \\
8 \mathrm{pc} \text { in radius }\end{array}$ & $120 \pm 30^{c}$ & -12.1 \\
\hline
\end{tabular}

Notwithstanding the difficulties of this problem, it is desirable to measure $\sigma$ in a larger number of nuclei, in particular in galaxies which are radio sources, and then to compare the values of $\sigma$ in active and passive galaxies.

\section{Gas, Dust and Infrared Emission}

Data have recently become available in two areas which are extremely important for understanding the energy production in nuclei of galaxies: (1) correlations between the presence of a small radio source in the nucleus and the presence of emission lines in the optical spectrum, (2) measurement of infrared fluxes from nuclei. These are examined below.

\subsection{Presence of emission lines in the optical SPeCtra of NUClei Which have a} SMALL RADIO SOURCE; PHYSICAL CONDITIONS OF THE IONIZED GAS

Comparison of optical and radio properties of galactic nuclei shows that there exists a correlation between the presence of a small radio source in the nucleus and the 
presence of emission lines in the optical spectrum of the nucleus. This correlation holds both for elliptical galaxies (Disney and Cromwell, 1971; Ekers and Ekers, 1973) and for spiral galaxies (Alloin, 1973).

Tables IIIa and IIIb summarize the data relative to the proportions of nuclei with radio emission and optical line emission for elliptical and spiral galaxies respectively. The galaxies used for these statistics are within $100 \mathrm{Mpc}$ of the Earth (taking $\mathrm{H}=100 \mathrm{~km} \mathrm{~s}^{-1} \mathrm{Mpc}^{-1}$ ). The data on the emission lines in the optical spectra are from Humason et al. (1956).

TABLE IIIa

Optical line emission in the nuclei of elliptical galaxies ${ }^{\mathrm{a}}$

\begin{tabular}{lrc}
\hline Radio Characteristics & Total number & $\begin{array}{l}\text { Number with } \\
\text { [O II] 3727 }\end{array}$ \\
\hline Galaxies with a radio source less than 6" in nucleus & 12 & 8 \\
Galaxies with extended sources & 7 & 0 \\
Galaxies not detected & 172 & 37 \\
\hline & &
\end{tabular}

TABLE IIIb

Optical line emission in the nuclei of spiral galaxies ${ }^{\mathrm{b}}$

\begin{tabular}{lll}
\hline $\begin{array}{l}\text { Type, and \% of galaxies of that type } \\
\text { in the sample }\end{array}$ & $\begin{array}{l}\text { Nuclei with a } \\
\text { small central } \\
\text { radio source }\end{array}$ & $\begin{array}{l}\text { Nuclei without } \\
\text { small central } \\
\text { sources }\end{array}$ \\
\hline $\mathrm{Sa} \quad 17 \%$ & $37 \%$ & $63 \%$ \\
$\mathrm{Sb} \quad 32 \%$ & $53 \%$ & $47 \%$ \\
$\mathrm{Sc} \quad 51 \%$ & $32 \%$ & $68 \%$ \\
$\begin{array}{l}\text { Presence of emission } \\
\text { lines in the spectra } \\
\text { of the nuclei, for all } \\
\text { types of spirals }\end{array}$ & $93 \%$ & $67 \%$ \\
\hline & & \\
\hline
\end{tabular}

Both tables show that emission lines in the optical spectrum occur more often in nuclei which have a central radio source than in nuclei without a central radio source.

The sample of elliptical galaxies analyzed by Ekers and Ekers is formed from galaxies observed by Humason et al. (1956). The radio observations were all carried out with the same instrument, and the criterion of radio structure (although necessarily arbitrary) according to which a galaxy is classified as having a small radio source is precisely defined: the central source must be smaller than 6". However, as was noted by Ekers and Ekers, even within 6" there is a large variety of radio sizes and structures. The sample analyzed by Alloin is made of spiral galaxies observed by Lequeux or by Wade, who used other criteria for defining a small central source (see Alloin, 1973), 
and as a result this sample of spiral galaxies is less homogeneous than the sample of Ekers and Ekers.

At present, only in a very few galaxies has the gas in the central region been analyzed in detail and these galaxies have radio sources in their nuclei. Therefore it is not possible to compare the physical conditions of the gas in galaxies with small central sources and without central sources.

The major uncertainty in our knowledge of the physical conditions of the gas in nuclei of galaxies comes from the fact that, although there is indirect evidence for the presence of various regions with different electron temperatures and densities and degrees of ionization, these regions cannot be resolved spatially with a ground-based telescope; as a consequence the line intensities which are measured result from the contributions of various regions where the gas has not necessarily the same physical conditions.

The physical conditions and abundances of the gas in the nuclei of M81, M51 and a few other relatively nearby large spiral galaxies with small central radio sources have been analyzed by Peimbert (1968), Alloin (1973) and Warner (1973). The major conclusions of these analyses can be summarized as follows:

(i) Since the intensity ratios of the lines of $\mathrm{O}_{\mathrm{I}}, \mathrm{O}_{\mathrm{II}}, \mathrm{O}_{\mathrm{III}}$ are not compatible with collisional ionization, it is concluded that the ionization must be radiative. The number of main sequence hot stars at $40000 \mathrm{~K}$ and $10 R_{\odot}$ which would be necessary to maintain the ionization is about 40 in M81 and M51 (Peimbert, 1968); in all the nuclei considered, the contribution of the flux emitted by these hot stars to the total flux of the nucleus would not exceed a few percent at $\lambda \sim 3500 \AA$ and therefore cannot be detected.

The $\left[\mathrm{O}_{\mathrm{I}}\right] /\left[\mathrm{O}_{\mathrm{II}}\right]$ line intensity ratio is considerably higher than in normal $\mathrm{H} \mathrm{II}$ regions, therefore it has been suggested that a substantial fraction of the ionization in the nuclei of these galaxies is due to hard UV radiation (Bergeron and Souffrin, 1971). The line intensity ratios calculated by Bergeron and Souffrin are in fair agreement with the observations, the largest disagreement occurring in the case of He I $\lambda 5876 / \mathrm{H} \alpha$ for which the predicted value seems a little too strong as compared with the upper limits observed by Peimbert and Spinrad (1970) in M51 and M81. Other ionization mechanisms like cloud collisions suggested by Peimbert (1973) should be investigated before the problem of the ionization in these nuclei can be considered settled.

(ii) Nitrogen is found to be overabundant by a factor 6 (with an uncertainty by a factor 2). This overabundance of $\mathrm{N}$ provides the most likely explanation for the rapid change of the line intensity ratio $\mathrm{H} \alpha /[\mathrm{N}$ II $] \lambda 6584$ from the value $\approx 3$ in the spiral arms to a value $\leqslant 1$ in the nucleus.

iii) There are large fluctuations of the electron density. The emitting gas is clumped in small clouds or filaments, the clumpiness factor being of the order of $10^{-4}$. There is also a gradual decrease of the electron density from the nucleus outward, on a scale of $5^{\prime \prime}$ (Warner, 1973).

That galaxies with compact nuclear radio sources have optical spectra with emission 
lines more often than do galaxies without central sources is a fact which is not understood at the present and raises interesting questions on which one can comment only in a very speculative way: Are the stars which are necessary to keep the gas ionized normally present in the nucleus, or has the onset of the radio source teen accompanied by a small outburst of star formation? Is the gas which is revealed by the emission lines normally present in the nuclei, or has it gathered or been released at the epoch the radio source started? The situation is further complicated by the fact that there exist a few galaxies with compact radio sources which apparently have only a very small amount of ionized gas and where the presence of non-thermal optical radiation is demonstrated by UV excess, variations, or linear polarization of the optical continuum. An example is B2 $1652+39$ (4C 39.49) which is an elliptical galaxy with a compact central radio source (Ekers, 1973). The nucleus is very bright in the optical range, and linear polarization of $2 \%$ has been measured by Kinman (1973). Emission lines can hardly be seen on the optical spectrum; the redshift measured from the $\mathrm{H}$ and $\mathrm{K}$ lines is 0.034 . Another example of such an elliptical galaxy is $3 \mathrm{C} 371$. Perhaps the objects like BL Lac are extreme cases of such nuclei which emit optical non-thermal radiation but have extremely weak emission lines.

\subsection{INFRARED EMISSION}

Infrared emission at $10 \mu$ has now been detected in 47 galaxies (Rieke and Low, 1972a). The total span of infrared luminosity at $10 \mu$ is $10^{6}$. The weakest of all the galaxies detected are M31, Maffei 1 and the galactic centre. The next in increasing luminosity are at least 50 times brighter than these galaxies of the Local Group. Most of the non-Seyfert galaxies detected by Rieke and Low have optical spectra with intense narrow emission lines, like NGC 7714 for example, whose spectrum in the $\mathrm{H} x$ region is shown in Figure 2. The non-Seyfert galaxies are, in general, weaker at $10 \mu$ than the Seyfert galaxies; yet there are a few exceptions like NGC 1614 (see Figure 2). This galaxy is the fourth brightest galaxy observed at $10 \mu$ and is 40 times brighter than NGC 4151. The emission lines in NGC 1614 are definitely narrower than in Seyfert-type spectra - even considering the wing of $\mathrm{H} \alpha$ extending down to [N $\left.{ }_{11}\right] \lambda 6548$, which is very probably caused by gas flowing out from the nucleus (Ulrich, 1972b).

The spectra from $2 \mu$ to $25 \mu$ have been measured for several of the galaxies with large apparent infrared luminosities (Rieke and Low, 1972a). The galaxies with Seyfert-type or active nuclei usually have a steep straight spectrum in the $\log f_{v}$ vs $\log v$ diagram. Flattening of the spectrum at $\lambda<3 \mu$ occurs in several cases and is readily explained by the contribution from cool stars in the vicinity of the nucleus. Some of the galaxies which are intrinsically weak infrared emitters have spectra which differ markedly from a steep straight spectrum, as can be seen in Figure 3.

\subsubsection{NGC 253 and NGC 3034 (M82)}

In each of these two galaxies the region emitting the $10 \mu$ flux is extended. In NGC 3034 , the $10 \mu$ source is elongated along the equatorial plane with dimensions $25^{\prime \prime} \times 8$ " or $400 \times 120 \mathrm{pc}$, and the radio source at $1420 \mathrm{MHz}$ is also elongated along the plane 


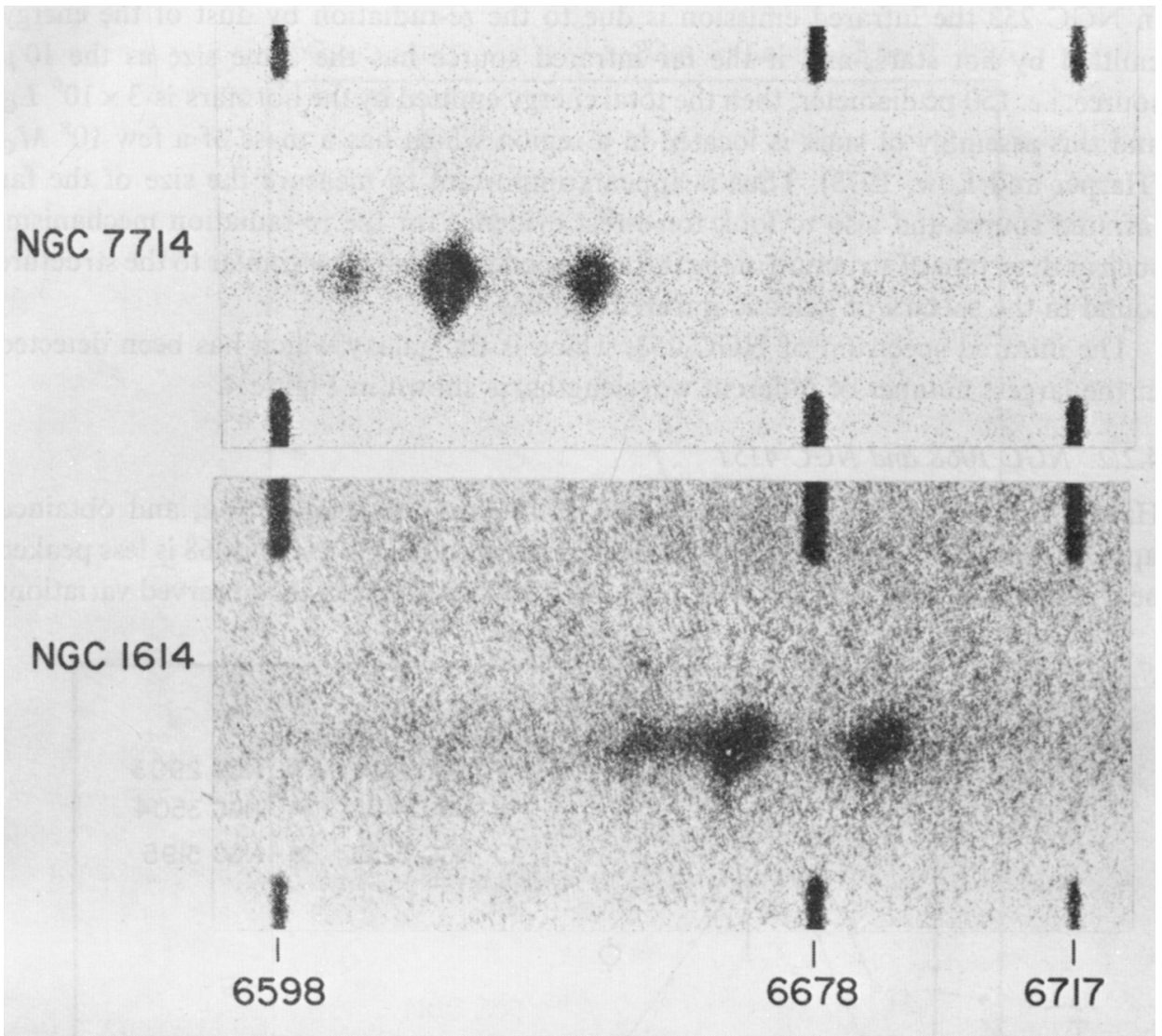

Fig. 2. $28 \AA \mathrm{mm}^{-1}$ spectrograms of NGC 7714 and NGC 1614 in the region of $\mathrm{H} \alpha$ and [N II] $\lambda \lambda 6548,6584$. The $10 \mu$-fluxes emitted by these galaxies have been measured by Rieke and Low (1972a) and are respectively two times and forty times more intense than the $10 \mu$-flux emitted by NGC 4151 .

with dimensions a little less than $30^{\prime \prime} \times 20^{\prime \prime}$ (see Kleinmann and Low, 1970). In NGC 253 , the core of the $10 \mu$ source is about $10^{\prime \prime}$ or $150 \mathrm{pc}$ in diameter and coincides in position and shape with the central radio source observed at $2695 \mathrm{MHz}$ (Becklin et al., 1973). M82 and NGC 253 have been detected at wavelengths between $27 \mu$ and $125 \mu$ (Harper and Low, 1973) with an airborne telescope $30 \mathrm{~cm}$ in diameter; the angular resolution of these observations is $2^{\prime}$ as compared to $5^{\prime \prime}$ for $\lambda<20 \mu$ and to $1^{\prime}$ for the ground-based observations at $350 \mu$. At $350 \mu$, Rieke et al. (1973) detected NGC 253 and set an upper limit on the flux from NGC 3034. These far-infrared data show that the energy distributions of NGC 253 and NGC 3034 have a very pronounced maximum near $150 \mu$. If the infrared emission in NGC 253 and NGC 3034 originated from a single synchrotron source, the value of the magnetic field causing synchrotron self-absorption longward of $200 \mu$ would be extremely high because of the large size of the source. This type of argument suggests that in these two galaxies the infrared flux is produced by re-radiation from dust particles. On the other hand, if 
in NGC 253 the infrared emission is due to the re-radiation by dust of the energy emitted by hot stars, and if the far infrared source has the same size as the $10 \mu$ source, i.e. $150 \mathrm{pc}$ diameter, then the total energy emitted by the hot stars is $3 \times 10^{9} L_{\odot}$ and this assembly of stars is located in a region which has a mass of a few $10^{8} M_{\odot}$ (Harper and Low, 1973). Thus it appears important to measure the size of the far infrared source and also to look for direct evidence for the re-radiation mechanism, such as detection of structure in the infrared spectrum near $10 \mu$ similar to the structure found in the spectra of galactic infrared sources.

The infrared spectrum of NGC 253, which is the galaxy which has been detected at the largest number of different wavelengths, is shown in Figure 4.

\subsection{2. $N G C 1068$ and NGC 4151}

Harper and Low (1973) searched for NGC 1068 at $100 \mu$ and $350 \mu$, and obtained upper limits on the fluxes which indicate that the spectrum of NGC 1068 is less peaked near $200 \mu$ than the spectra of NGC 253 and NGC 3034. From the observed variations

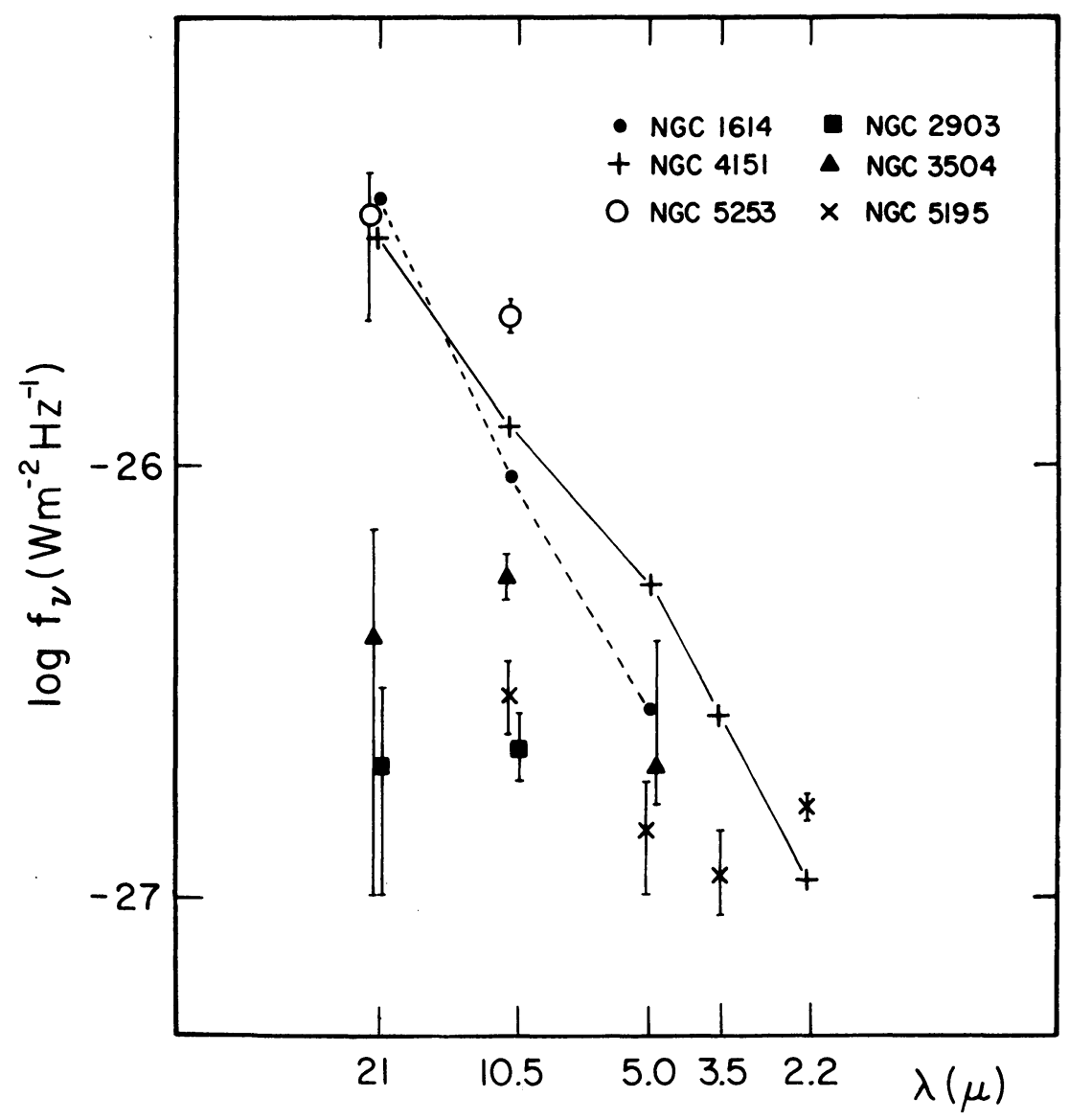

Fig. 3. Infrared spectra of a few of the galaxies observed by Rieke and Low, 1972a. 


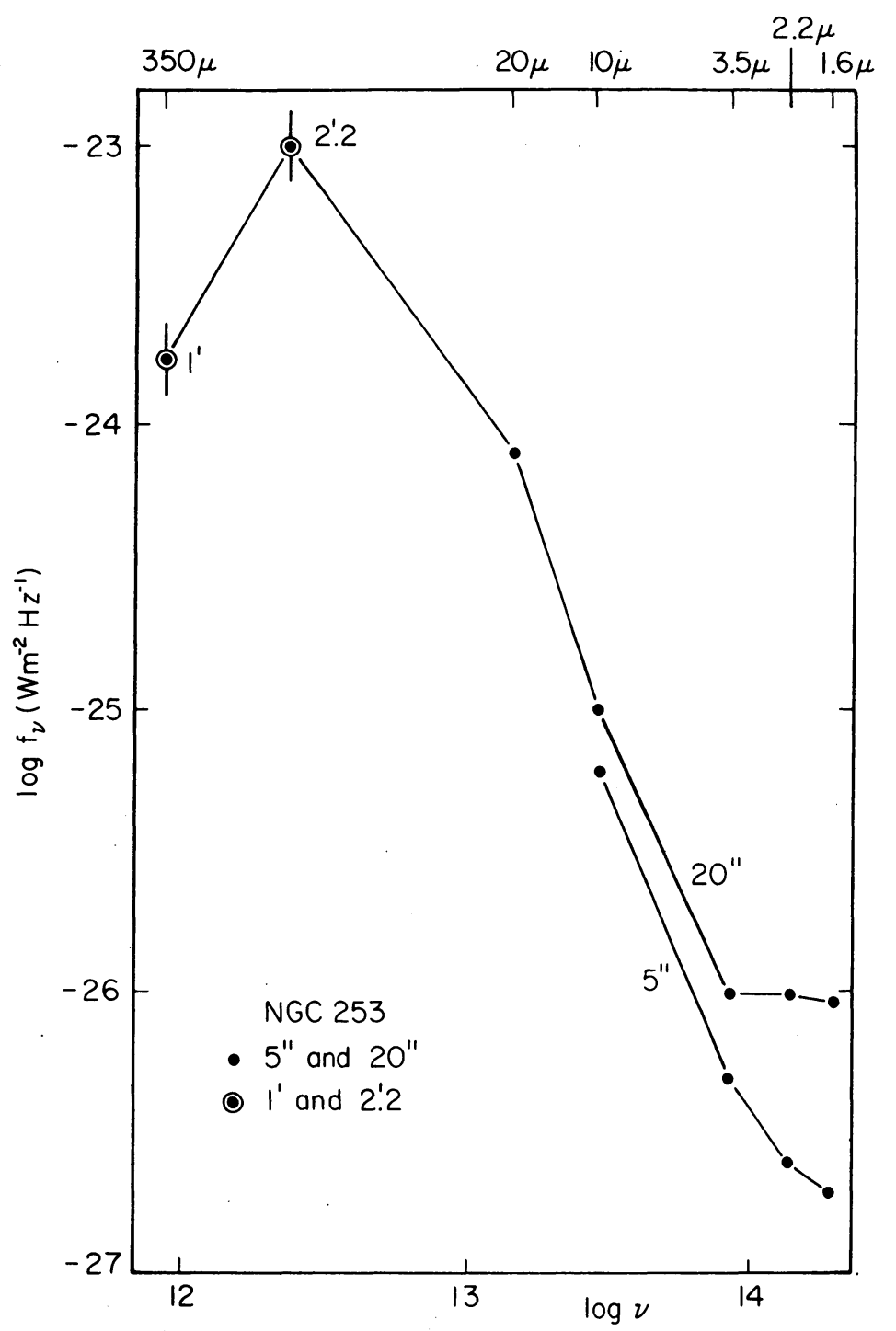

Fig. 4. Infrared spectrum of NGC 253 between 1.6 and $350 \mu$. Note that the entrance aperture was not the same for all the wavelengths at which the flux was measured. Data are from Becklin et al. (1973), from Harper and Low (1973) and from Harper et al. (1972).

of the $10 \mu$ flux of NGC 1068, Rieke and Low (1972b) have concluded that in this galaxy the infrared emission is of non-thermal origin. However, the variability of the $10 \mu$ flux is seriously questioned by Stein et al. (1974) who monitored NGC 1068 for over three years, occasionally at the same time as Rieke and Low, and it is not clear at present whether the $10 \mu$ flux of NGC 1068 shows variations large enough to rule out its origin as re-radiation by dust.

Stein et al. (1974) also observed the spectrum of NGC 1068 between $8 \mu$ and $13 \mu$ 
with a resolution $\Delta \lambda / \lambda \sim 0.05$; the spectrum is structureless, in contrast to the spectra observed for galactic sources in which the source of energy is interpreted as thermal re-radiation by grains. The discovery of a structureless spectrum between 8 and $13 \mu$ does not entirely rule out the re-radiation mechanism, although it seems difficult to build a model with dust particles having properties similar to those found in the infrared galactic sources and, at the same time, having an infrared spectrum withcut structure.

In NGC 4151, Rieke and Low (1972b) and Stein et al. (1974) found some evidence for changes of the $10 \mu$ flux by $50 \%$ on a time scale of approximately 100 days, and therefore most of the infrared flux is of non-thermal origin.

Our present knowledge on the infrared emission from nuclei of galaxies can te summarized as follows:

(i) Seyfert-type galaxies are the galaxies which have the highest luminosity at $10 \mu$. In NGC 4151, the variations of the $10 \mu$ flux strongly suggest that the infrared emission is of non-thermal origin.

(ii) In the two nearby galaxies NGC 253 and NGC 3034, which are at a distance of $\sim 3 \mathrm{Mpc}$, the $10 \mu$ source is extended with typical dimensions of $200 \mathrm{pc}$. The far infrared spectrum has a sharply peaked maximum at $200 \mu$. Because of the large dimension of the infrared source and of the sharp decrease of the flux longward of $200 \mu$, emission by a single synchrotron source can be rejected in favour of re-radiation by dust as the source of the infrared luminosity.

(iii) A number of galaxies, mostly spirals, with intense and narrow emission lines have been detected at $10 \mu$.

\section{Active Nuclei}

Generally speaking, the nucleus of a galaxy is considered to be active if a detectable fraction of the electromagnetic spectrum cannot be explained by stellar radiation, radiation by interstellar gas at $T_{\mathrm{e}}<2 \times 10^{4} \mathrm{~K}$ and re-radiation by dust particles. Nuclei where expansion motions are present are also considered active : the expansion motions are understood as the result of radiation pressure from a source of ultraviolet continuum on dense gas close to this source, and thus expansion motions are indirect evidence of the presence in the recent past of a source of ultraviolet continuum probably of non-thermal origin. Depending upon the most striking features of their spectra or their morphology, active nuclei have different names, e.g. Seyfert-type nuclei, N-galaxies, but the classification of active galaxies into various groups is not discussed here. It is the view of the author that the current terminology is temporarily useful but not satisfactory.

Recent observations of three properties of active nuclei are discussed below: $\mathrm{X}$-ray emission, profiles of the permitted lines and variations of the line intensities, structure of the forbidden lines.

\subsection{X- AND GAMMA-RAY EMISSION}

The only nuclear region which has so far been detected with certainty by several 
groups of observers as a source of gamma radiation is the Galactic Centre. However, it is not yet clear whether the radiation comes from several discrete sources or from one diffuse source (Fazio 1972).

Two other sources of gamma-radiation which may be associated with galaxies are:

(i) a source in the direction of the Seyfert galaxy 3C 120 (Volobuev et al., 1971) and

(ii) a source in the direction of AP Lib (PKS 1514-24) which is an object of BL Lac type (Frye et al., 1971). The angular resolution of the gamma-ray observations is poor, with error boxes of several degrees in size, but 3C 120 and AP Lib seem to be the most likely optical identifications for these two sources. 3C 120 was detected in late 1968 at a time of intense activity at centimetric wavelengths; the source in the direction of AP Lib is variable and was seen in December 1969 but not in February 1969.

In the hard $\mathrm{X}$-ray range, about $50 \mathrm{keV}$, the only galaxy detected with certainty is NGC 5128. The spectrum between 1 and $180 \mathrm{keV}$ can be fitted with a power law $v^{-\alpha}$ with $\alpha$ between 1.45 and 2.0 (Lampton et al., 1972).

UHURU satellite observations have provided a great wealth of data between 2 and $10 \mathrm{keV}$ (Giacconi et al., 1972). The spectra of the three individual extragalactic objects NGC 4151, NGC 5128 and 3C 273 display a most interesting feature, viz. a definite cut-off for energy lower than $\sim 5 \mathrm{keV}$. Observed upper limits on the angular sizes of the X-ray sources associated with NGC 4151 and NGC 5128 are 15' and 10' respectively, corresponding to linear sizes of 50 and $15 \mathrm{kpc}$. An indirect argument based on the presence of the cut-off in the spectrum suggests that the X-ray source is substantially smaller than the upper limit on the size obtained by the observations (Giacconi, 1973). In NGC 4151, the large cut-off corresponds to a column density of absorbing material of $10^{23}$ atom $\mathrm{cm}^{-2}$; if the X-ray source were as large as the observed upper limit on the size, the total mass of the absorbing gas would be $\sim 3.5 \times 10^{11} M_{\odot}$. Such a large mass of absorbing gas is quite unlikely and a more reasonable configuration is one in which the X-ray source is in the nucleus of the galaxy and surrounded by a relatively small volume of dense gas. Such a model has been discussed in detail by Tucker et al. (1973) in the case of NGC 5128.

The X-ray source detected in M31 is comparatively weak and the X-ray spectrum has not yet been measured. The intensity is consistent with the emission being the sum of several discrete sources as is the case in the Magellanic Clouds. The peculiar galaxy M82 has also been detected in the range $2-8 \mathrm{keV}$. The number of counts per second is about the same as from the X-ray source in the direction of $3 \mathrm{C} 273$, and slightly below the count rate of NGC 4151 (Giacconi et al., 1973).

\subsection{Profiles of the Permitted lines; VARiations of LiNe intensities}

In the spectra of many Seyfert nuclei the permitted lines are very broad with widths of $100 \AA$ or more, whereas the forbidden lines have widths less than $20 \AA$. This fact is regarded as evidence for the existence of at least 2 regions: one with an electron density less than $10^{6} \mathrm{~cm}^{-3}$ which emits forbidden and permitted lines, and another region with $N_{\mathrm{e}}>10^{6} \mathrm{~cm}^{-3}$ which emits permitted lines only and produces the broad 
component of these lines. Because of the large value of the electron density in the latter region, electron scattering could be an important broadening agent of the permitted lines. This question has been investigated by Weymann (1970) and by Mathis (1970) who found that electron scattering could in some cases explain the profiles of the permitted lines. In the case of NGC 4151, Weymann showed that a small region with $N_{\mathrm{c}}=2.5 \times 10^{9} \mathrm{~cm}^{-3}$ and a dimension of only 0.005 pc would emit the observed $\mathrm{H} \alpha$ intensity. NGC 4151 is the best known case of a Seyfert-type spectrum where the hydrogen lines have a sharp intense core and smooth symmetrical wings reminiscent of the calculated profile caused by electron scattering.

The observed profiles of the permitted lines available at the time of Weymann's and of Mathis' investigations were not known with an accuracy high enough to allow meaningful comparisons with theoretical profiles produced by electron scattering. Since then, relatively accurate profiles with spectral resolutions of 2 to $5 \AA$ have been published by Anderson (1971) for NGC 5548, by Ulrich (1972a) for NGC 5548 and NGC 3516 and by Shields et al. (1972) for 3C 120. The observed profiles are either asymmetric or show structures or irregularities which rule out electron scattering as the main broadening agent. It is concluded that in these nuclei the profiles of the permitted lines are caused by mass motions. Less detailed observations of other nuclei are also consistent with this conclusion.

In the spectra of some nuclei, the short wavelength side of the hydrogen lines is more intense than the long wavelength side (e.g. NGC 5548, NGC 1614), or the hydrogen lines have a blueshifted component (e.g. 3C 227, 3C 390.3, Markarian 6). This feature can be explained by the presence of dense gas flowing out of the nucleus in two opposite directions, with the gas flowing away from us being partly hidden by material which is optically thick; this material could be the dense gas itself or some other component of the nucleus. It must be noted that if the dense gas itself is optically thick then only a lower limit on its mass can be calculated from the absolute intensities of the hydrogen lines.

The mass necessary to emit an $\mathrm{H} \beta$ line of absolute intensity $\sim 10^{41} \mathrm{erg} \mathrm{s}^{-1}-$ which is typical of a fairly weak Seyfert nucleus - is approximately $15 M_{\odot}$ if the electron density is $10^{8} \mathrm{~cm}^{-3}$. It is easy to see that if the high velocities indicated by the width of the hydrogen lines represent expansion motions in a single cloud of this mass, observable weakening of the hydrogen lines should occur on a time scale of 3-30 yr. But no such rapid variations are expected if the gas is clumped in small clouds which have high relative velocities but small internal velocity dispersion, a situation reminiscent of the gaseous filaments of the Crab Nebula.

It has been shown that, if the non-thermal optical continuum is extrapolated into the ultraviolet with a power-law spectrum, there is enough energy to accelerate the dense gas to the observed velocities by radiation pressure (Mushotzky et al., 1972; Shields et al., 1972).

We have seen above that mass transfer can produce line intensity variations, but only for permitted lines because of the small dimension of the single cloud from which these lines can in principle originate. Variations of the intensities of the permitted 
and the forbidden lines can also be caused by variations of the photo-ionizing flux, provided this flux comes from a central source. Bahcall et al. (1972) showed that, in some cases, changes in line intensities can occur over times that are small compared with the light travel-time across the nebula.

Rapid variations of line intensities in the nuclei of the Seyfert galaxies NGC 4151, 3516 and 1068 have been reported by Cherepashchuk and Lyutyi (1973). The observations consisted of measuring the fluxes received through a $150 \AA$ pass-band centred on $H \alpha$, the fluxes received through the same pass band at $\pm 300 \AA$ from $H \alpha$, and the $U$ magnitudes.

The amplitudes of variations of the lines reach $34 \%$ for NGC 3516 . Variations were observed with time scales of 15 days for NGC 4151 and 35 days for NGC 3516. The variations of flux in the band centred on $\mathrm{H} \alpha$ were attributed by the authors to $\mathrm{H} \alpha$ only but this band included the two forbidden lines [N $\mathrm{II}] \lambda \lambda 6548,6584$.

Cherepashchuk and Lyutyi observed variations of the $U$ magnitude which resemble the variations of the $\mathrm{H} \alpha$ line but with a time delay of 15 to 30 days between the variations of the line and the variations of the $U$ magnitude, the variations in $U$ magnitude always preceding the variations of the line (Figure 5a).

Variations of the profiles of the blended emission lines $\mathrm{H} \alpha+\left[\mathrm{N} \mathrm{II}_{\mathrm{II}} \lambda \lambda 6548,6584\right.$ of NGC 1068 have been reported by Eilek et al. (1973) during a time interval which was

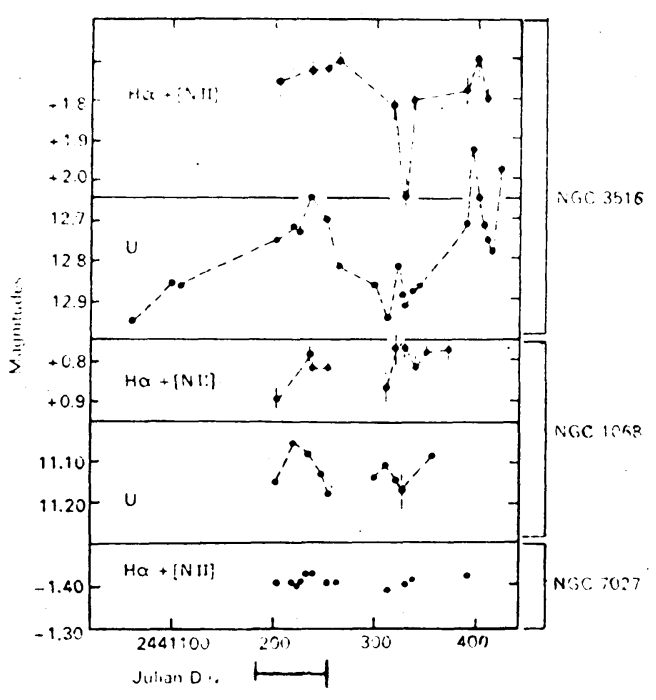

(a)

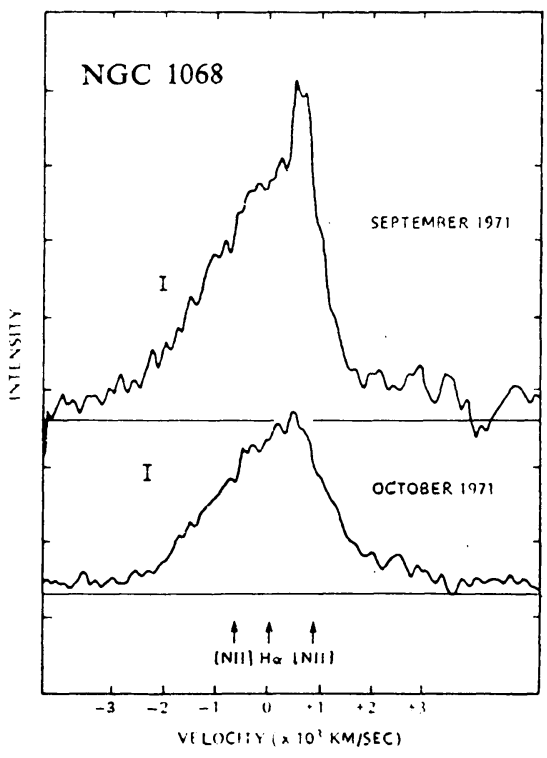

(b)

Fig. 5. Variations of the intensities and of the profiles of emission lines in nearby Seyfert galaxies; Figure 5a is from Cherepashchuk and Lyuti (1973), Figure 5b is from Eilek et al. (1973). The horizontal bar below Figure 5a represents the epoch of the observations of NGC 1068

by Eilek et al. (1973). 
also covered by Cherepashchuk and Lyutyi. The observations were made between August 28 and October 23, 1971 with an isocon television camera. Comparison of the profiles of the blend $\mathrm{H} \alpha+\left[\mathrm{N}_{\text {II }}\right] \lambda \lambda 6548,6584$ shows that in September the [N II] $\lambda \lambda 6584$ peak was unusually strong relative to $\mathrm{H} \alpha$ but by October it was back to its level of August (Figure 5b).

Prior to the observations of Cherepashchuk and Lyutyi, and of Eilek et al., variations of the intensity of the hydrogen lines had already been reported to have occurred in NGC 1566 (Pastoriza and Gerola, 1970), NGC 3516 (Collin-Souffrin et al., 1973) and IC 450 (Markarian 6) (Khachikian and Weedman, 1971; Notni et al., 1973). These variations of line intensities can be readily understood as due to the variations of the photoionizing flux. Note, however, the scanner observations of 3C 120 by Shields et al. (1972) covering a period of $3 \mathrm{yr}$, during which the continuum varied by $20 \%$ while the emission lines did not vary.

It thus appears that the amplitudes and time scales of the variations of the line intensities triggered by the variations of the continuum flux are different from object to object and probably also from epoch to epoch in a given nucleus.

Observations which are most needed are monitoring of the profiles of the lines (for example $\mathrm{H} \alpha$ and [N $\mathrm{NI}] \lambda \lambda 6548,6584$ or $\mathrm{H} \beta$ and [O III] 4959, 5007), and also simultaneous monitorings of the absolute line intensities and of the continuum flux. They will provide data necessary to make progress on the determination of important parameters of the emission line regions such as linear dimensions, clumpiness factors of the gas, etc.

How the high-velocity gas affects the physical conditions of the ambient less-dense gas is a question which has not yet been investigated.

\subsection{StRucture of THE FORBIDden LiNeS}

The structures of the forbidden lines in the spectra of NGC 7469, NGC 4151 and Markarian 78 have recently been investigated. In NGC 7469, the motions indicated by the forbidden lines are interpreted by Anderson (1973) as due to rotation. If this interpretation is correct the mass of the central region $400 \mathrm{pc}$ in radius is about $10^{9} M_{\odot}$.

In NGC 4151 the four clouds found by Ulrich (1973) are roughly aligned in position angle $40^{\circ}$ which is close to the direction of the major axis determined by $21-\mathrm{cm}$ observations (Davies, 1973); therefore the motions of these clouds may plausibly be caused by rotation. The most prominent feature of NGC 4151 on direct plates is an ellipsoidal ring, $4^{\prime} \times 8^{\prime}$, whose major axis is perpendicular to the major axis determined by Davies. Prior to the $21-\mathrm{cm}$ observations this prominent feature was assumed to be circular and was used to determine the orientation in space of the equatorial plane of the galaxy. If the determination of the major axis from the $21-\mathrm{cm}$ observations is valid, which is quite likely, then the orientation and shape of this ellipsoidal feature becomes very puzzling and it would be of interest to observe its motions.

Clouds of ionized gas have also been found in the inner region of Markarian 78 (Adams, 1973); they are roughly aligned along the major axis. It is not known at the 
present if their velocity difference is due to the rotation of the galaxy or to expansion motions.

The mass of the nuclear region of an active galaxy, determined from the measurement of intense forbidden lines which show structures or large velocity dispersions, should be regarded with caution because the question arises as to whether the features which are measured truly represent the rotation of the stellar component in which lies most of the mass of the central region.

\section{What Fraction of Galactic Nuclei are Active?}

We have seen earlier that in the optical range of wavelengths the signs of activity of a galaxy are the presence of expansion motions or emission of non-thermal continuum flux. Evidently it is very difficult to search for these signs in a statistically meaningful fashion. This is in contrast with the situation in the radio range where searches for compact nuclear sources can be made down to well-defined limits of flux and of angular dimension.

The set of galaxies which is the best known in the optical range is that formed by the nearest galaxies. Let us consider the galaxies which were observed by Humason et al. (1956), which are not members of the Virgo Cluster nor of the Local Group, and which have $V_{\text {corrected }}<1000 \mathrm{~km} \mathrm{~s}^{-1}$. This defines a set of 126 galaxies among which 7 (i.e. $5 \%$ ) have nuclei which are active* and can be recognized as such by their optical properties alone. The values of $5 \%$ is a lower limit to the fraction of active nuclei, because not all the galaxies of this set have been thoroughly searched for signs of activity in the optical range.

Since this set contains very few elliptical galaxies, this discussion of the fraction of 'optically active nuclei' is relevant to spiral galaxies only; the presence of dwarf galaxies in this set is ignored. Finally, it is of interest to note that among this set of 126 galaxies, 16 have small radio sources in their nuclei (Ekers, 1973), and among these 16 galaxies are all the galaxies in which activity has been observed in the optical range.

\section{Acknowledgements}

I thank my colleagues for helpful discussions on nuclei of galaxies, in particular R. Ekers and M. Peimbert.

This work was supported in part through grant GP-25220 from the National Science Foundation.

* These 7 galaxies are:

NGC 253 (Demoulin and Burbidge, 1970)

NGC 3034 (Lynds and Sandage, 1963)

NGC 4051 (Seyfert, 1943)

NGC 4151 (Seyfert, 1943)

NGC 4258 (Courtès and Cruvellier, 1961)

NGC 4736 (Chincarini and Walker, 1967)

NGC 5194 (Burbidge and Burbidge, 1964) 


\section{References}

Adams, T. F.: 1973, Astrophys. J. 179, 417.

Alloin, D.: 1973, Astron. Astrophys. 27, 433.

Alloin, D., Andrillat, Y., and Souffrin, S.: 1971, Astron. Astrophys. 10, 401.

Anderson, K. S.: 1971, Astrophys. J. 169, 449.

Anderson, K. S.: 1973, Astrophys. J. 182, 369.

Andrillat, Y. and Souffrin, S.: 1971, Astron. Astrophys. 11, 286.

Andrillat, Y., Souffrin, S., and Alloin, D.: 1972, Astron. Astrophys. 19, 405.

Bahcall, J. N., Kozlovsky, Ben-Zion, and Salpeter, E. E.: 1972, Astrophys. J. $171,467$.

Baldwin, R. J., Danziger, I. J., Frogel, J. A., and Persson, S. E.: 1973, Astrophys. Letters $14,1$.

Becklin, E. E., Fomalont, E. B., and Neugebauer, G.: 1973, Astrophys. J. Letters, 181, L27.

Bergeron, J. and Souffrin, S.: 1971, Astron. Astrophys. 14, 167.

Blanc-Vaziaga, M-J., Cayrel, G., and Cayrel, R.: 1973, Astrophys. J. 180, 871.

Burbidge, G. R.: 1970, Ann. Rev. Astron. Astrophys. 8, 369.

Burbidge, E. M. and Burbidge, G. R.: 1964, Astrophys. J. 140, 1445.

Cherepashchuk, A. M. and Lyutyi, V. M.: 1973, Astrophys. Letters 13, 165.

Chincarini, G. and Walker, M. F.: 1967, Astrophys. J. 147, 407.

Code, A. D., Welch, G. A., and Page, T. L.: 1972, in A. D. Code (ed.), Scientific Results from OAO-2, NASA SP-310, Washington, p. 559.

Collin-Souffrin, S., Alloin, D., and Andrillat, Y.: 1973, Astron. Astrophys. 22, 343.

Courtès, G. and Cruvellier, P.: 1961, Compt. Rend. Acad. Sci. Paris 253, 218.

Cromwell, R. and Weymann, R.: 1970, Astrophys. J. Letters 159, 147.

Danielson, R. E., Light, E. A., Schwarzschild, M., and Tomasko, M. G.: 1971, Bull. Am. Astron. Soc. 4, 230.

Davies, R. D.: 1973, Monthly Notices Roy. Astron. Soc. 161, 25 P.

Demoulin, M.-H. and Burbidge, E. M.: 1970, Astrophys. J. 159, 799.

Disney, M. J. and Cromwell, R. H.: 1971, Astrophys. J. Letters 164, 35.

Eilek, J. A., Auman, J. R., Ulrych, T. J., and Walker, G. A. H.: 1973, Astrophys. J. 182, 363.

Ekers, R. D. and Ekers, J. A.: 1973, Astron. Astrophys. 24, 247.

Ekers, R. D.: 1973, private communication.

Faber, S. M.: 1972, Astron. Astrophys. 20, 361.

Faber, S. M.: 1973, Astrophys. J. 179, 731.

Fazio, G. G.: 1972, in H. Bradt and R. Giacconi (eds.), 'X- and Gamma-Ray Astronomy', IAU' Symp. 55, 303.

Frye, G. M., Albats, P. A., Zych, A. D., Staib, J. A., Hopper, V. D., Rawlinson, W. R., and Thomas, J. A.: 1971, Nature 233, 466.

Giacconi, R.: 1973, Phys. Today, May, p. 38.

Giacconi, R., Murray, S., Gursky, H., Kellogg, E., Schreier, E., and Tananbaum, H.: 1972, Astrophys. J. 178, 281.

Giacconi, R., Murray, S., Gursky, H., Kellogg, E., Schreier, E., Matilsky, T., Koch, D., and Tananbaum, H.: 1973, Astrophys. J. 188, 667.

Harper, D. A. and Low, F. J.: 1973, Astrophys. J. Letters 182, 89.

Harper, D. A., Low, F. J., Rieke, G., and Armstrong, K. R.: 1972, Astrophys. J. Letters $177,21$.

Humason, M. L., Mayall, N. U., and Sandage, A. R.: 1956, Astron. J. 61, 97.

Johnson, H. M.: 1961, Astrophys. J. 133, 309.

Khachikian, E. Ye. and Weedman, D. W.: 1971, Astrophys. J. Letters 164, 109.

Kinman, T. D.: 1973, private communication.

Kleinmann, D. E. and Low, F. J.: 1970, Astrophys. J. Letters 161, 203.

Lallemand, A., Duchesne, M., and Walker, M. F.: 1960, Publ. Astron. Soc. Pacific 72, 76.

Lampton,'M., Margon, B., Bowyer, S., Mahoney, W., and Anderson, K.: 1972, Astrophys. J. Letters 171, 45 .

Lynds, C. R. and Sandage, A. R.: 1963, Astrophys. J. 137, 1005.

Mathis, J. S.: 1970, Astrophys. J. 162, 761.

McClure, R. D.: 1969, Astron. J. 74, 50.

McClure, R. D. and van den Bergh, S.: 1968, Astron. J. 73, 313. 
Morton, D. C. and Chevalier, R. A.: 1973, Astrophys. J. 179, 55.

Morton, D. C. and Thuan, Trinh, X.: 1973, Astrophys. J. 180, 705.

Mushotzky, R. F., Solomon, P. M., and Strittmatter, P. A.: 1972, Astrophys. J. 174, 7.

Notni, P., Khachikian, E. Ye., Butslov, M. M., and Gevorkian, G. T.: 1973, Astrofizika 9, 39.

Pastoriza, M. and Gerola, H.: 1970, Astrophys. Letters 6, 155.

Peimbert, M.: 1968, Astrophys. J. 154, 33.

Peimbert, M.: 1973, Private Communication.

Peimbert, M. and Spinrad, H.: 1970, Astrophys. J. 159, 809.

Poveda, A.: 1958, Bol. Obs. Tonantzintla Tacubaya No. 17.

Richstone, D. and Sargent, W. L. W.: 1972, Astrophys. J. 176, 91.

Rieke, G. H. and Low, F. J.: 1972a, Astrophys. J. Letters 176, 95.

Rieke, G. H. and Low, F. J.: 1972b, Astrophys. J. Letters 177, 115.

Rieke, G. H., Harper, D. A., Low, F. J., and Armstrong, K. R.: Astrophys. J. Letters 183 L67.

Rubin, V. C. and Ford, W. K.: 1971, Astrophys. J. 170, 25.

Rubin, V. C., Ford, W. K., and Krishna Kumar, C.: 1973, Astrophys. J. 181, 61.

Sandage, A. R., Becklin, E. E., and Neugebauer, G.: 1969, Astrophys. J. 157, 55.

Schwarzschild, M.: 1971, Bull. Am. Astron. Soc. 3, 243.

Seyfert, C. K.: 1943, Astrophys. J. 97, 28.

Shields; G. A., Oke, J. B., and Sargent, W. L. W.: 1972, Astrophys. J. 176, 75.

Spinrad, H.: 1972, Astrophys. J, 177, 285.

Spinrad, H. and Taylor, B. J.: 1971, Astrophys. J. Suppl. 2, 445.

Spinrad, H., Gunn, J. E., Taylor, B. J., McClure, R. D., and Young, J. W.: 1971, Astrophys. J. 164, 11. Spinrad, H., Smith, H. E., and Taylor, D. J.: 1972, Astrophys. J. 175, 649.

Stein, W. A., Gillett, F. C., and Merrill, K. M.: 1973, Astrophys. J. 187, 213.

Strom, S. E., Strom, K. M., and Carbon, D. F.: 1971, Astron. Astrophys. 12, 177.

Tinsley, B.: 1973, Astrophys. J. Letters 184, 41.

Tinsley, B. M. and Spinrad, H.: 1971, Astrophys. Space Sci. 12, 118.

Tucker, W., Kellogg, E., Gursky, H., Giacconi, R., and Tananbaum, H.: 1973, Astrophys. J. 180, 715.

Ulrich, M.-H.: 1971, Astrophys. J. Letters, 165, 61.

Ulrich, M.-H.: 1972a, Astrophys. J. 174, 483.

Ulrich, M.-H.: 1972b, Astrophys. J. 178, 113.

Ulrich, M.-H.: 1973, Astrophys. J. 181, 51.

Volobuev, S. A., Galper, A. M., Kirillov-Ugryumov, V. G., Luchkov, B. I., and Ozerov, Yu. V.: 1971, Astron. Zh. 48, 1105; Soviet Astron. 15, 879, 1972.

Wampler, E. J.: 1968, Astrophys. J. Letters 154, L53.

Wampler, E. J.: 1971, Astrophys. J. 164, 1.

Warner, J. W.: Astrophys. J. 186, 21.

Weymann, R. J.: 1970, Astrophys. J. 160, 31.

Whitford, A. E.: 1972, Bull. Am. Astron. Soc. 4, 230.

Wood, D. B.: 1966, Astrophys. J. 145, 36.

\section{DISCUSSION}

Freeman: Relevant to the last part of this talk are variations observed in NGC 1566 (also noted by the Cordoba group). In 1962 this object had a Seyfert-like nuclear spectrum with $\mathrm{H} \beta 2000 \mathrm{~km} \mathrm{~s}^{-1}$ wide. In $1968 \mathrm{H} \beta$ was double with two components $800 \mathrm{~km} \mathrm{~s}^{-1}$ apart, one at the same redshift as the other emission lines, and the other to the red. Now there is only one sharp $\mathrm{H} \beta$ line and one would not call NGC 1566 a Seyfert system any more.

Danziger: I have a comment which may be relevant to the previous discussion and more particularly the report by Ron Ekers of the presence of a non-thermal radio source in the nucleus of M81. Mrs Jean Goad, a graduate student at Harvard, is studying the dynamics of the nuclear region of M81 and has found evidence for a significant non-circular component in the velocity of the gas which could be interpreted as ejection of matter.

Kerr: Outflow of gas in the central regions has been discussed for our own and other galaxies. Recent work has shown that the $3 \mathrm{kpc}$ arm and other features observed in the Galaxy can be interpreted as a dispersion ring, in which the gas is moving in gravitationally-stable elliptical orbits. 
Under these circumstances, there is apparent outward motion but there is no need to assume the existence of energetic explosions. Similar phenomena may occur in other galaxies.

van der Kruit: Fits of 'dispersion rings' to the non-circular motions in central regions of galaxies have been made in particular in our Galaxy (Shane, W. W.: Astron. Astrophys. 16, 118, 1972; Simonson, S. C. and Mader, G. L.: Bull. Am. Astron. Soc. 4, 266, 1973) and in M51 (Tully, R. B.: Univ. of Maryland, Ph.D. Thesis, 1972). In this respect it must be noted that:

(i) the only unambiguous determinations of the direction of non-circular motions (against $\mathrm{Sgr} \mathrm{A}$ in our Galaxy and along the minor axis in M51) give expansions. This is also true for other galaxies such as M31.

(ii) The solutions for the geometry of such dispersion rings are, in both cases, those where the velocities in the line of sight are the same as for a field of expanding motions and thus those where we cannot decide between the two alternatives. This seems rather suspicious.

Davies: I have two comments about NGC 4151 further to my discussion in Monthly Notices Roy. Astron. Soc. 161, 25P, 1973. Firstly, Mr A. Murray has taken photographs for me with the Isaac Newton Telescope, and isodensity tracings by $\mathrm{Dr} \mathrm{C}$. Fraser show that the central regions at a mean radius of $5^{\prime \prime}$ have an axial ratio of 1.06 , the position angle of the major axis being $35^{\circ}$. This is similar to the $\mathrm{H}$ I dynamical major axis at $26^{\circ}+3^{\circ}$. Secondly, the velocities found in the inner $6^{\prime \prime}$ by $\mathrm{Dr}$ Ulrich are in the same sense as the $\mathrm{H}_{\mathrm{I}}$ rotational velocities in the outer part of the galaxy, implying that the inner parts of the galaxy are also rotating. The mass inside a radius of $2^{\prime \prime}(100 \mathrm{pc})$ would then be $6 \times 10^{9} M_{\odot}$, assuming an inclination of about $30^{\circ}$, and gravitational equilibrium. 